Kragujevac Journal of Mathematics

Volume 45(1) (2021), Pages 63-73.

\title{
ON PERFECT CO-ANNIHILATING-IDEAL GRAPH OF A COMMUTATIVE ARTINIAN RING
}

\author{
S. M. SAADAT MIRGHADIM ${ }^{1}$, M. J. NIKMEHR ${ }^{2}$, AND R. NIKANDISH ${ }^{3}$
}

\begin{abstract}
Let $R$ be a commutative ring with identity. The co-annihilating-ideal graph of $R$, denoted by $A_{R}$, is a graph whose vertex set is the set of all nonzero proper ideals of $R$ and two distinct vertices $I$ and $J$ are adjacent whenever $\operatorname{Ann}(I) \cap \operatorname{Ann}(J)=(0)$. In this paper, we characterize all Artinian rings for which both of the graphs $A_{R}$ and $\overline{A_{R}}$ (the complement of $A_{R}$ ), are chordal. Moreover, all Artinian rings whose $A_{R}$ (and thus $\overline{A_{R}}$ ) is perfect are characterized.
\end{abstract}

\section{INTRODUCTION}

Assigning a graph to a ring gives us the ability to translate algebraic properties of rings into graph-theoretic language and vice versa. It leads to arising interesting algebraic and combinatorics problems. Therefore, the study of graphs associated with rings has attracted many researches. There are a lot of papers which apply combinatorial methods to obtain algebraic results in ring theory; for instance see $[2,3,5,6,10,11]$ and $[12]$.

Throughout this paper, all rings are assumed to be commutative with identity. We denote by $Z(R), \operatorname{Max}(R), \operatorname{Nil}(R)$ and $J(R)$ the set of all zero-divisor elements of $R$, the set of all maximal ideals of $R$, the set of all nilpotent elements of $R$ and jacobson radical of $R$, respectively. We call an ideal $I$ of $R$, an annihilating-ideal if there exists $r \in R \backslash\{0\}$ such that $I r=(0)$. The set of all annihilating-ideals of $R$ is denote by $A(R)$. Let $I$ be an ideal of $R$. We denote by $A(I)$ the set of all ideals of $R$ contained in $I$. The ring $R$ is said to be reduced if it has no non-zero nilpotent element. For every ideal $I$ of $R$, we denote the annihilator of $I$ by $\operatorname{Ann}(I)$. We let $A^{*}=A \backslash\{0\}$. For any undefined notation or terminology in ring theory, we refer the reader to $[4,7]$.

Key words and phrases. Co-annihilating-ideal graph, perfect graph, chordal graph.

2010 Mathematics Subject Classification. Primary: 05C19. Secondary: 05C75, 05C25.

DOI 10.46793/KgJMat2101.063M

Received: January 03, 2018.

Accepted: September 06, 2018. 
We use the standard terminology of graphs following [13]. Let $G=(V, E)$ be a graph, where $V=V(G)$ is the set of vertices and $E=E(G)$ is the set of edges. By $\bar{G}$, we mean the complement graph of $G$. We write $u-v$, to denote an edge with ends $u, v$. A graph $H=\left(V_{0}, E_{0}\right)$ is called a subgraph of $G$ if $V_{0} \subseteq V$ and $E_{0} \subseteq E$. Moreover, $H$ is called an induced subgraph by $V_{0}$, denoted by $G\left[V_{0}\right]$, if $V_{0} \subseteq V$ and $E_{0}=\left\{\{u, v\} \in E \mid u, v \in V_{0}\right\}$. Also $G$ is called a null graph if it has no edge. A complete graph of $n$ vertices is denoted by $K_{n}$. An $n$-part graph is one whose vertex set can be partitioned into $n$ subsets, so that no edge has both ends in any one subset. A complete n-partite graph is an n-part graph such that every pair of graph vertices in the $n$ sets are adjacent. In a graph $G$, a vertex $x$ is isolated, if no vertices of $G$ is adjacent to $x$. Let $G_{1}$ and $G_{2}$ be two disjoint graphs. The join of $G_{1}$ and $G_{2}$, denoted by $G_{1} \vee G_{2}$, is a graph with the vertex set $V\left(G_{1} \vee G_{2}\right)=V\left(G_{1}\right) \cup V\left(G_{2}\right)$ and edge set $E\left(G_{1} \vee G_{2}\right)=E\left(G_{1}\right) \cup E\left(G_{2}\right) \cup\left\{u v \mid u \in V\left(G_{1}\right), v \in V\left(G_{2}\right)\right\}$. For a graph $G$, $S \subseteq V(G)$ is called a clique if the subgraph induced on $S$ is complete. The number of vertices in the largest clique of graph $G$ is called the clique number of $G$ and is often denoted by $\omega(G)$. For a graph $G$, let $\chi(G)$ denote the chromatic number of $G$, i.e., the minimal number of colors which can be assigned to the vertices of $G$ in such a way that every two adjacent vertices have different colors. Clearly, for every graph $G, \omega(G) \leq \chi(G)$. A graph $G$ is said to be weakly perfect if $\omega(G)=\chi(G)$. A perfect graph $G$ is a graph in which every induced subgraph is weakly perfect. A chord of a cycle $C$ is an edge which is not in $C$ but has both its endvertices in $C$. A graph $G$ is chordal if every cycle of length at least 4 has a chord.

Let $R$ be a commutative ring with identity. The co-annihilating-ideal graph of $R$, denoted by $A_{R}$, is a graph whose vertex set is the set of all non-zero proper ideals of $R$ and two distinct vertices $I$ and $J$ are adjacent whenever $\operatorname{Ann}(I) \cap \operatorname{Ann}(J)=(0)$. This graph was first introduced and studied in [1] and many interesting properties of this graph were explored by the authors. In [1, Theorem 17], it was proved $A_{R}$ is a weakly perfect graph, if $R$ is an Artinian ring. In this paper, we continue study the perfectness of $A_{R}$. Indeed, we characterize all Artinian rings for which both of the graphs $A_{R}$ and $\overline{A_{R}}$, are chordal. Moreover, all Artinian rings whose $A_{R}$ is perfect are given.

\section{When $A_{R}$ AND $\overline{A_{R}}$ ARE ChORdal?}

In this section, we characterize all Artinian rings $R$, for which $A_{R}$ and $\overline{A_{R}}$ are chordal. We begin with the following lemmas.

Lemma 2.1. Let $R$ be an Artinian ring. Then there exists a positive integer $n$ such that $R \cong R_{1} \times \cdots \times R_{n}$, where $R_{i}$ is an Artinian local ring, for every $1 \leq i \leq n$.

Proof. See [4, Theorem 8.7].

Lemma 2.2. Let $R$ be an Artinian ring and $I$ be a non-zero ideal of $R$. Then $I$ is a nilpotent ideal of $R$ if and only if $I$ is an isolated vertex in $A_{R}$. 
Proof. Assume that $I$ is a non-zero nilpotent ideal of $R$. First, we show that $\operatorname{Ann}(I)$ is an essential ideal of $R$. Suppose to the contrary, there exists an ideal $J$ such that $J \cap \operatorname{Ann}(I)=(0)$. Thus $K I \neq(0)$, for every $K \subseteq J$. Obviously, $K I \subseteq J$ and so $(K I) I=K I^{2} \neq(0)$. By continuing this procedure, $K I^{n} \neq 0$, for every positive integer $n$, a contradiction. Hence $\operatorname{Ann}(I)$ is an essential ideal of $R$ and so $\operatorname{Ann}(I) \cap \operatorname{Ann}(J) \neq(0)$, for every $J \in A(R)^{*}$. Therefore, $I$ is an isolated vertex in $A_{R}$.

Conversely, suppose that $I$ is an isolated vertex in $A_{R}$. If $I$ is not a nilpotent ideal of $R$, then $I \nsubseteq J(R)$, i.e, there exists $\mathfrak{m} \in \operatorname{Max}(R)$ such that $I+\mathfrak{m}=R$, and so $I$ is adjacent to $\mathfrak{m}$, a contradiction. Thus $I$ is a nilpotent ideal of $R$.

Next we need to study the structure of $A_{R}$, where $R$ is an Artinian ring with at most two maximal ideals.

Theorem 2.1. Let $R$ be an Artinian ring. Then the following statements are equivalent:

(1) $|\operatorname{Max}(R)|=1$;

(2) $A_{R}=\overline{K_{n}}$, where $n=\left|A(R)^{*}\right|$.

Proof. (1) $\Rightarrow(2)$ Since $R$ is an Artinian local ring, every ideal of $A(R)^{*}$ is a nilpotent ideal of $R$ and thus by Lemma $2.2, A_{R}$ is a null graph.

$(2) \Rightarrow(1)$ is obtained by Lemma 2.2 .

Theorem 2.2. Let $R$ be an Artinian ring. Then the following statements are equivalent:

(1) $|\operatorname{Max}(R)|=2$;

(2) $A_{R}=\overline{K_{n_{1}}}+K_{n_{2}, n_{3}}$, where $n_{1}=\left|A(\operatorname{Nil}(R))^{*}\right|, n_{2}=\left|A\left(\mathfrak{m}_{1}\right)^{*}\right|-n_{1}, n_{3}=$ $\left|A\left(\mathfrak{m}_{2}\right)^{*}\right|-n_{1}$ and $\mathfrak{m}_{1}, \mathfrak{m}_{2} \in \operatorname{Max}(R)$.

Proof. (1) $\Rightarrow(2)$ Let $\operatorname{Max}(R)=\left\{\mathfrak{m}_{1}, \mathfrak{m}_{2}\right\}$. Since $\mathfrak{m}_{1} \cap \mathfrak{m}_{2}=\operatorname{Nil}(R)$, Lemma 2.2 implies that $A_{R}\left[A(\operatorname{Nil}(R))^{*}\right]$ is a null graph. Let $A=\left\{I \in A\left(\mathfrak{m}_{1}\right) \backslash A(\operatorname{Nil}(R))\right\}$ and $B=\left\{I \in A\left(\mathfrak{m}_{2}\right) \backslash A(\operatorname{Nil}(R))\right\}$. If $I \in A$ and $J \in B$, then $I+J=R$, and thus $I$ is adjacent to $J$. Moreover, $A_{R}[A]$ and $A_{R}[B]$ are null graphs. This means that $A_{R}[A \cup B]=K_{|A|,|B|}$. Since $A \cup B \cup A(\operatorname{Nil}(R))^{*}=A(R)^{*}$, we deduce that $A_{R}=\overline{K_{n_{1}}}+K_{n_{2}, n_{3}}$, where $n_{1}=\left|A(\operatorname{Nil}(R))^{*}\right|, n_{2}=\left|A\left(\mathfrak{m}_{1}\right)^{*}\right|-n_{1}, n_{3}=\left|A\left(\mathfrak{m}_{2}\right)^{*}\right|-n_{1}$ and $\mathfrak{m}_{1}, \mathfrak{m}_{2} \in \operatorname{Max}(R)$.

$(2) \Rightarrow(1)$ By Theorem 2.1, $|\operatorname{Max}(R)| \geq 2$. If $|\operatorname{Max}(R)| \geq 3$, then $A_{R}$ has a cycle of length 3 , as $A_{R}[\operatorname{Max}(R)]$ is a complete graph, a contradiction. Thus $|\operatorname{Max}(R)|=2$.

We are now in a position to characterize all Artinian rings for which both of the graphs $A_{R}$ and $\overline{A_{R}}$ are chordal.

Theorem 2.3. Let $R$ be an Artinian ring. Then

(1) $A_{R}$ is chordal if and only if one of the following statements holds:

(i) $R$ is local;

(ii) $R \cong F \times S$, where $F$ is a field and $S$ is local; 
(iii) $R \cong F_{1} \times F_{2} \times F_{3}$, where $F_{i}$ is a field for every $1 \leq i \leq 3$;

(2) $\overline{A_{R}}$ is chordal if and only if $|\operatorname{Max}(R)| \leq 3$.

Proof. (1) Let $A_{R}$ be chordal. First we show that $|\operatorname{Max}(R)| \leq 3$. If $|\operatorname{Max}(R)| \geq 4$, then Figure 1 is a cycle of length 4 ,

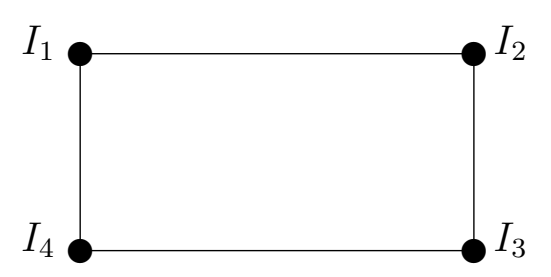

Figure 1. A cycle of length 4 in $A_{R}$

where

$$
\begin{aligned}
& I_{1}=(0) \times R_{2} \times R_{3} \times(0) \times R_{5} \times \cdots \times R_{n}, \\
& I_{2}=R_{1} \times(0) \times(0) \times R_{4} \times R_{5} \times \cdots \times R_{n}, \\
& I_{3}=R_{1} \times R_{2} \times R_{3} \times(0) \times R_{5} \times \cdots \times R_{n}, \\
& I_{4}=R_{1} \times(0) \times R_{3} \times R_{4} \times R_{5} \times \cdots \times R_{n} .
\end{aligned}
$$

Thus $|\operatorname{Max}(R)| \leq 3$. If $|\operatorname{Max}(R)|=3$, then $R \cong R_{1} \times R_{2} \times R_{3}$, where $R_{i}$ is an Artinian local ring, for every $1 \leq i \leq n$. If $R_{1}$ is not field, then consider $I \in A\left(\operatorname{Nil}\left(R_{1}\right)\right)^{*}$ and thus Figure 2 is a cycle of length 4 ,

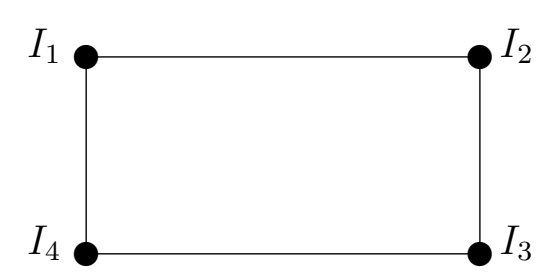

Figure 2. A cycle of length 4 in $A_{R}$

where

$$
\begin{aligned}
& I_{1}=R_{1} \times(0) \times(0), \\
& I_{2}=(0) \times R_{2} \times R_{3}, \\
& I_{3}=R_{1} \times R_{2} \times(0), \\
& I_{4}=I \times R_{2} \times R_{3} .
\end{aligned}
$$

Hence $R_{1}$ is a field. Similarly, $R_{2}$ and $R_{3}$ are fields. Let $|\operatorname{Max}(R)|=2$. Then $R \cong R_{1} \times R_{2}$, where $R_{i}$ is an Artinian local ring, for every $1 \leq i \leq 2$. We show that 
one of the rings $R_{1}$ and $R_{2}$ is a field. If $I, J$ are non-zero proper ideals of $R_{1}$ and $R_{2}$, respectively, then Figure 3 is a cycle of length 4, where

$$
\begin{aligned}
& I_{1}=I \times R_{2}, \\
& I_{2}=R_{1} \times J, \\
& I_{3}=(0) \times R_{2}, \\
& I_{4}=R_{1} \times(0) .
\end{aligned}
$$

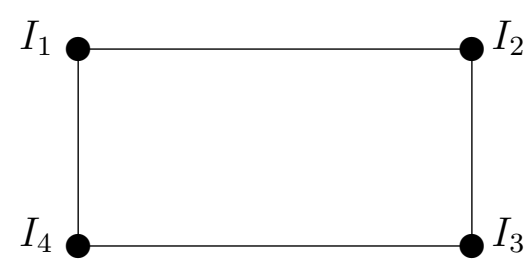

Figure 3. A cycle of length 4 in $A_{R}$

This means that one of the rings $R_{1}$ and $R_{2}$ is a field. Thus in this case $R \cong F \times S$, where $F$ is a field and $S$ is local. Clearly, if $|\operatorname{Max}(R)|=1, R$ is local.

Conversely, suppose that one of the conditions (i), (ii), (ii) is satisfied. Condition (i) implies that $A_{R}$ is a null graph by Theorem 2.1, and thus $A_{R}$ is chordal. If (ii) holds, then by Theorem 2.2, $A_{R}=\overline{K_{n}}+K_{1, n+1}$ where $n=\left|A(\operatorname{Nil}(R))^{*}\right|$. This implies that $A_{R}$ is chordal. If (iii) holds, then Figure 4 shows that $A_{R}$ is chordal where

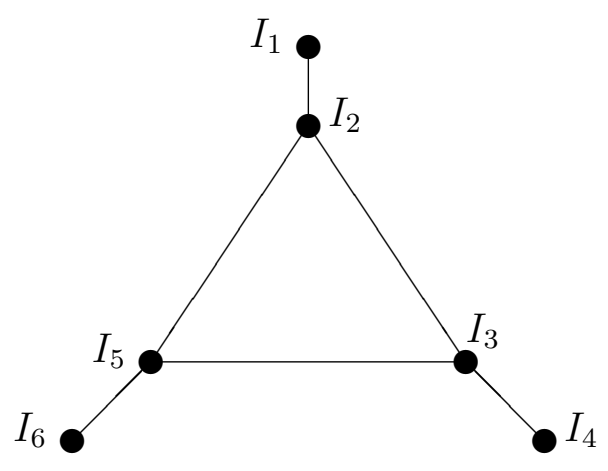

FIGURE 4. $A_{F_{1} \times F_{2} \times F_{3}}$

$$
\begin{aligned}
& I_{1}=(0) \times(0) \times F_{3}, \\
& I_{2}=F_{1} \times F_{2} \times(0), \\
& I_{3}=F_{1} \times(0) \times F_{3}, \\
& I_{4}=(0) \times F_{2} \times(0), \\
& I_{5}=(0) \times F_{2} \times F_{3},
\end{aligned}
$$




$$
I_{6}=F_{1} \times(0) \times(0) .
$$

(2) First suppose that $\overline{A_{R}}$ is chordal. If $|\operatorname{Max}(R)| \geq 4$, then we put

$$
\begin{aligned}
& I_{1}=(0) \times R_{2} \times R_{3} \times(0) \times R_{5} \times \cdots \times R_{n}, \\
& I_{2}=(0) \times R_{2} \times(0) \times R_{4} \times R_{5} \times \cdots \times R_{n}, \\
& I_{3}=R_{1} \times(0) \times(0) \times R_{4} \times R_{5} \times \cdots \times R_{n}, \\
& I_{4}=R_{1} \times(0) \times R_{3} \times(0) \times R_{5} \times \cdots \times R_{n} .
\end{aligned}
$$

Now, it is not hard to see that $I_{1}-I_{2}-I_{3}-I_{4}-I_{1}$ is a cycle of length 4 , a contradiction. Thus $|\operatorname{Max}(R)| \leq 3$.

Conversely, suppose that $|\operatorname{Max}(R)| \leq 3$. We show that $\overline{A_{R}}$ is chordal. To see this, we consider the following cases.

Case 1. $|\operatorname{Max}(R)|=1$. In this case, $R$ is local and thus by Theorem $2.1, \overline{A_{R}}$ is a complete graph. Hence $\overline{A_{R}}$ is chordal.

Case 2. $|\operatorname{Max}(R)|=2$. By Theorem 2.2, $\overline{A_{R}}=K_{n_{1}} \bigvee\left(K_{n_{2}}+K_{n_{3}}\right)$, where $n_{1}=$ $\left|A(\operatorname{Nil}(R))^{*}\right|, n_{2}=\left|A\left(\mathfrak{m}_{1}\right)^{*}\right|-n_{1}, n_{3}=\left|A\left(\mathfrak{m}_{2}\right)^{*}\right|-n_{1}$ and $\mathfrak{m}_{1}, \mathfrak{m}_{2} \in \operatorname{Max}(R)$. Thus every cycle is a triangle, i.e, $\overline{A_{R}}$ is chordal.

Case 3. $|\operatorname{Max}(R)|=3$. In this case, $R \cong R_{1} \times R_{2} \times R_{3}$. Let $I_{i}$ be an ideal of $R_{i}$, for every $1 \leq i \leq 3$. Suppose that

$$
\begin{aligned}
& A_{1}=\left\{I_{1} \times I_{2} \times I_{3} \mid I_{i} \subseteq \operatorname{Nil}\left(R_{i}\right), \text { for } i=1,2,3\right\} \backslash\{(0) \times(0) \times(0)\} \\
& A_{2}=\left\{R_{1} \times I_{2} \times I_{3} \mid I_{i} \subseteq \operatorname{Nil}\left(R_{i}\right), \text { for } i=2,3\right\} \\
& A_{3}=\left\{I_{1} \times R_{2} \times I_{3} \mid I_{i} \subseteq \operatorname{Nil}\left(R_{i}\right), \text { for } i=1,3\right\} \\
& A_{4}=\left\{I_{1} \times I_{2} \times R_{3} \mid I_{i} \subseteq \operatorname{Nil}\left(R_{i}\right), \text { for } i=1,2\right\} \\
& B_{1}=\left\{R_{1} \times R_{2} \times I_{3} \mid I_{3} \subseteq \operatorname{Nil}\left(R_{3}\right)\right\} \\
& B_{2}=\left\{R_{1} \times I_{2} \times R_{3} \mid I_{2} \subseteq \operatorname{Nil}\left(R_{2}\right)\right\} \\
& B_{3}=\left\{I_{1} \times R_{2} \times R_{3} \mid I_{1} \subseteq \operatorname{Nil}\left(R_{1}\right)\right\}
\end{aligned}
$$

Let $A=\cup_{i=1}^{4} A_{i}$ and $B=\cup_{i=1}^{3} B_{i}$. One may check that $A \cap B=\emptyset$ and $V\left(\overline{A_{R}}\right)=A \cup B$ and so $\{A, B\}$ is a partition of $V\left(\overline{A_{R}}\right)$. We claim that $\overline{A_{R}}$ contains no induced cycle of length at least 4 . Assume to the contrary, $a_{1}-a_{2}-\cdots-a_{n}-a_{1}$ is an induced cycle of length at least 4 in $\overline{A_{R}}$. We show that

$$
\left\{a_{1}, a_{2}, \ldots, a_{n}\right\} \cap B_{1}=\emptyset .
$$

Suppose to the contrary (and with no loss of generality), $a_{1} \in B_{1}$. Thus $a_{1}=$ $R_{1} \times R_{2} \times I_{3}$, where $I_{3} \subseteq \operatorname{Nil}\left(R_{3}\right)$. Since $a_{2}$ and $a_{n}$ are adjacent to $a_{1}$, we conclude that the third components of $a_{2}$ and $a_{n}$ must be nilpotent ideals of $R_{3}$. This implies that $a_{2}$ and $a_{n}$ are adjacent, a contradiction. Hence,

$$
\left\{a_{1}, a_{2}, \ldots, a_{n}\right\} \cap B_{1}=\emptyset .
$$

Similarly,

$$
\left\{a_{1}, a_{2}, \ldots, a_{n}\right\} \cap B_{2}=\left\{a_{1}, a_{2}, \ldots, a_{n}\right\} \cap B_{3}=\emptyset .
$$


This means that

$$
\left\{a_{1}, a_{2}, \ldots, a_{n}\right\} \subseteq A .
$$

But this contradicts the fact that $\overline{A_{R}}[A]$ is a complete graph, and so $\overline{A_{R}}$ contains no induced cycle of length at least 4 . Thus $\overline{A_{R}}$ is chordal.

\section{When $A_{R}$ IS Perfect?}

In this section, we characterize all Artinian rings rings $R$ whose $A_{R}$ is Perfect. First, we need two celebrate results.

Theorem 3.1 (The Strong Perfect Graph Theorem [8]). A graph $G$ is perfect if and only if neither $G$ nor $\bar{G}$ contains an induced odd cycle of length at least 5 .

In light of Theorem 3.1, we have the following corollary.

Corollary 3.1. Let $G$ be a graph. Then the following statements hold.

(1) $G$ is a perfect graph if and only if $\bar{G}$ is a perfect graph.

(2) If $G$ is a complete bipartite graph, then $G$ is a perfect graph.

Theorem 3.2. [9] Every chordal graph is perfect.

Lemma 3.1. Let $n$ be a positive integer and $R \cong R_{1} \times \cdots \times R_{n}$, where $R_{i}$ is an Artinian ring for every $1 \leq i \leq n$. Let $I=I_{1} \times \cdots \times I_{n}, J=J_{1} \times \cdots \times J_{n}$ be two distinct ideals of $R$ and $n \geq 2$. Then $I-J$ is an edge of $A_{R}$ if and only if for every $1 \leq i \leq n, I_{i} \notin A\left(\mathrm{Nil}\left(R_{i}\right)\right)$ or $J_{i} \notin A\left(\mathrm{Nil}\left(R_{i}\right)\right)$.

Proof. Let $I-J$ be an edge of $A_{R}$. If there exists $1 \leq i \leq n$ such that $I_{i}, J_{i} \in$ $A\left(\operatorname{Nil}\left(R_{i}\right)\right)$, then by Lemma $2.2, \operatorname{Ann}\left(I_{i}\right) \cap \operatorname{Ann}\left(J_{i}\right) \neq(0)$. So if $0 \neq a_{i} \in \operatorname{Ann}\left(I_{i}\right) \cap$ $\operatorname{Ann}\left(J_{i}\right)$, then $(0) \times \cdots \times(0) \times R_{i} a_{i} \times(0) \times \cdots \times(0) \subseteq \operatorname{Ann}(I) \cap \operatorname{Ann}(J)$ and thus $I-J$ is not an edge of $A_{R}$, a contradiction.

Conversely, suppose that $I_{i} \notin A\left(\operatorname{Nil}\left(R_{i}\right)\right)$ or $J_{i} \notin A\left(\operatorname{Nil}\left(R_{i}\right)\right)$, for every $1 \leq i \leq n$. Thus $I_{i}=R_{i}$ or $J_{i}=R_{i}$, for every $1 \leq i \leq n$. This implies that $\operatorname{Ann}(I) \cap \operatorname{Ann}(J)=(0)$. Hence $I-J$ is an edge of $A_{R}$.

We are now in a position to state our main result in this paper.

Theorem 3.3. Let $R$ be an Artinian rings. Then $\overline{A_{R}}$ is a perfect graph if and only if $|\operatorname{Max}(R)| \leq 4$.

Proof. First suppose $\overline{A_{R}}$ is perfect. Since $R$ is an Artinian ring, there exists a positive integer $n=|\operatorname{Max}(R)|$ such that $R \cong R_{1} \times \cdots \times R_{n}$, where $R_{i}$ is an Artinian local ring, for every $1 \leq i \leq n$, by Lemma 2.1. If $n \geq 5$, then we put

$$
\begin{aligned}
& I_{1}=(0) \times R_{2} \times R_{3} \times(0) \times R_{5} \times R_{6} \times \cdots \times R_{n}, \\
& I_{2}=(0) \times R_{2} \times(0) \times R_{4} \times R_{5} \times R_{6} \times \cdots \times R_{n}, \\
& I_{3}=R_{1} \times(0) \times(0) \times R_{4} \times R_{5} \times R_{6} \times \cdots \times R_{n}, \\
& I_{4}=R_{1} \times(0) \times R_{3} \times R_{4} \times(0) \times R_{6} \times \cdots \times R_{n},
\end{aligned}
$$




$$
I_{5}=R_{1} \times R_{2} \times R_{3} \times(0) \times(0) \times R_{6} \times \cdots \times R_{n} .
$$

Then it is easily seen that

$$
I_{1}-I_{2}-I_{3}-I_{4}-I_{5}-I_{1}
$$

is a cycle of length 5 in $\overline{A_{R}}$, a contradiction (by Theorem 3.1). So $n \leq 4$.

Conversely, suppose that $|\operatorname{Max}(R)| \leq 4$. We show that $\overline{A_{R}}$ is a perfect graph. If $|\operatorname{Max}(R)| \leq 3$, then by part (2) of Theorem 2.3, $\overline{A_{R}}$ is chordal and thus by Theorem $3.2, \overline{A_{R}}$ is a perfect graph. Therefore, we need only to check the case $|\operatorname{Max}(R)|=4$. Let $R \cong R_{1} \times R_{2} \times R_{3} \times R_{4}$. We have the following claims.

Claim 1. $\overline{A_{R}}$ contains no induced odd cycle of length at least 5 . We consider the following partition for $V\left(\overline{A_{R}}\right)$ :

$$
\begin{aligned}
& A=\left\{I_{1} \times I_{2} \times I_{3} \times I_{4} \mid I_{i} \in A\left(R_{i}\right) \text { for every } 1 \leq i \leq 4 \text { and } I_{4} \in A\left(\operatorname{Nil}\left(R_{4}\right)\right)\right\} \\
& B=\left\{I_{1} \times I_{2} \times I_{3} \times R_{4} \mid I_{i} \in A\left(R_{i}\right) \text { for every } 1 \leq i \leq 3 \text { and } I_{3} \in A\left(\operatorname{Nil}\left(R_{3}\right)\right)\right\} \\
& C=\left\{I_{1} \times I_{2} \times R_{3} \times R_{4} \mid I_{i} \in A\left(R_{i}\right) \text { for every } 1 \leq i \leq 2 \text { and } I_{2} \in A\left(\operatorname{Nil}\left(R_{2}\right)\right)\right\} \\
& D=\left\{R_{1} \times I_{2} \times R_{3} \times R_{4}, I_{1} \times R_{2} \times R_{3} \times R_{4} \mid \text { for every } 1 \leq i \leq 2 I_{i} \in A\left(\operatorname{Nil}\left(R_{i}\right)\right)\right\}
\end{aligned}
$$

Now, assume to the contrary, $a_{1}-a_{2}-\cdots-a_{n}-a_{1}$ is an induced odd cycle of length at least 5 in $\overline{A_{R}}$. We consider the following cases.

Case 1. $\left\{a_{1}, a_{2}, \ldots, a_{n}\right\} \cap D=\emptyset$. Let $a_{i} \in\left\{a_{1}, a_{2}, \ldots, a_{n}\right\} \cap D$, for some $1 \leq i \leq n$. Then we can let $a_{i}=I_{1} \times R_{2} \times R_{3} \times R_{4}$ or $a_{i}=R_{1} \times I_{2} \times R_{3} \times R_{4}$. If $a_{i}=I_{1} \times R_{2} \times R_{3} \times R_{4}$, then the first components of $a_{i-1}$ and $a_{i+1}$ must be in $A\left(\operatorname{Nil}\left(R_{i}\right)\right)$ and $A\left(\operatorname{Nil}\left(R_{i}\right)\right)$, respectively. So by Lemma 3.1, $a_{i-1}$ is adjacent to $a_{i+1}$, a contradiction. Thus, $a_{i} \neq I_{1} \times R_{2} \times R_{3} \times R_{4}$. Similarly, $a_{i} \neq R_{1} \times I_{2} \times R_{3} \times R_{4}$. This means that $\left\{a_{1}, a_{2}, \ldots, a_{n}\right\} \cap D=\emptyset$.

Case 2. $\left\{a_{1}, a_{2}, \ldots, a_{n}\right\} \cap C=\emptyset$. First we show that $\left|\left\{a_{1}, a_{2}, \ldots, a_{n}\right\} \cap C\right| \leq 1$. Let $a, b \in\left\{a_{1}, a_{2}, \ldots, a_{n}\right\} \cap C$. Then we can easily check that if there exits $x \in V\left(\overline{A_{R}}\right)$ such that $\operatorname{Ann}(x) \cap \operatorname{Ann}(a) \neq(0)$, then $\operatorname{Ann}(x) \cap \operatorname{Ann}(b) \neq(0)$. This means that if $x$ is adjacent to $a$, then $x$ is adjacent to $b$, a contradiction. So $\left|\left\{a_{1}, a_{2}, \ldots, a_{n}\right\} \cap C\right| \leq 1$. This together with the fact that $\overline{A_{R}}[A]$ and $\overline{A_{R}}[B]$ are complete subgraphs, imply that $n=5$ and $\left|\left\{a_{1}, a_{2}, \ldots, a_{n}\right\} \cap B\right|=\left|\left\{a_{1}, a_{2}, \ldots, a_{n}\right\} \cap A\right|=2$. Hence $\mid\left\{a_{1}, a_{2}, \ldots, a_{n}\right\} \cap$ $C \mid=1$, and thus we can let $a \in\left\{a_{1}, a_{2}, \ldots, a_{n}\right\} \cap C$. Since $a$ is adjacent to all vertices of $B \backslash\left\{R_{1} \times R_{2} \times I_{3} \times R_{4} \mid I_{3} \subseteq \operatorname{Nil}\left(R_{3}\right)\right\}$ and $\overline{A_{R}}[B]$ is a complete subgraph, $a_{i} \in\left\{a_{1}, a_{2}, \ldots, a_{n}\right\} \cap\left\{R_{1} \times R_{2} \times I_{3} \times R_{4} \mid I_{3} \subseteq \operatorname{Nil}\left(R_{3}\right)\right\}$, for some $1 \leq i \leq n$. We can let $a_{i}=R_{1} \times R_{2} \times I_{3} \times R_{4}$. Since only one of the components of $a_{i}$ is a nilpotent ideal of $R_{i}$, by a similar argument to that of case 1 , we get a contradiction. Hence, $\left\{a_{1}, a_{2}, \ldots, a_{n}\right\} \cap C=\emptyset$.

By the above cases, $\left\{a_{1}, a_{2}, \ldots, a_{n}\right\} \subseteq A \cup B$, but this contradicts the fact $\overline{A_{R}}[A]$ and $\overline{A_{R}}[B]$ are complete graphs, and thus $\overline{A_{R}}$ contains no induced odd cycle of length at least 5 . 
Claim 2. $A_{R}$ contains no induced odd cycle of length at least 5 . We consider the following partition for $V\left(A_{R}\right)$ :

$$
\begin{aligned}
A_{1}= & \left\{I_{1} \times R_{2} \times R_{3} \times R_{4} \mid I_{1} \in A\left(\operatorname{Nil}\left(R_{1}\right)\right)\right\}, \\
A_{2}= & \left\{R_{1} \times I_{2} \times R_{3} \times R_{4} \mid I_{2} \in A\left(\operatorname{Nil}\left(R_{2}\right)\right)\right\}, \\
A_{3}= & \left\{R_{1} \times R_{2} \times I_{3} \times R_{4} \mid I_{3} \in A\left(\operatorname{Nil}\left(R_{3}\right)\right)\right\}, \\
A_{4}= & \left\{R_{1} \times R_{2} \times R_{3} \times I_{4} \mid I_{4} \in A\left(\operatorname{Nil}\left(R_{4}\right)\right)\right\}, \\
B_{1}= & \left\{I_{1} \times I_{2} \times R_{3} \times R_{4} \mid I_{1} \in A\left(\operatorname{Nil}\left(R_{1}\right)\right), I_{2} \in A\left(\operatorname{Nil}\left(R_{2}\right)\right)\right\}, \\
B_{2}= & \left\{R_{1} \times R_{2} \times I_{3} \times I_{4} \mid I_{3} \in A\left(\operatorname{Nil}\left(R_{3}\right)\right), I_{4} \in A\left(\operatorname{Nil}\left(R_{4}\right)\right)\right\}, \\
B_{3}= & \left\{I_{1} \times R_{2} \times I_{3} \times R_{4} \mid I_{1} \in A\left(\operatorname{Nil}\left(R_{1}\right)\right), I_{3} \in A\left(\operatorname{Nil}\left(R_{3}\right)\right)\right\}, \\
B_{4}= & \left\{R_{1} \times I_{2} \times R_{3} \times I_{4} \mid I_{2} \in A\left(\operatorname{Nil}\left(R_{2}\right)\right), I_{4} \in A\left(\operatorname{Nil}\left(R_{4}\right)\right)\right\}, \\
B_{5}= & \left\{I_{1} \times R_{2} \times R_{3} \times I_{4} \mid I_{1} \in A\left(\operatorname{Nil}\left(R_{1}\right)\right), I_{4} \in A\left(\operatorname{Nil}\left(R_{4}\right)\right)\right\}, \\
B_{6}= & \left\{R_{1} \times I_{2} \times I_{3} \times R_{4} \mid I_{2} \in A\left(\operatorname{Nil}\left(R_{2}\right)\right), I_{3} \in A\left(\operatorname{Nil}\left(R_{3}\right)\right)\right\}, \\
C_{1}= & \left\{R_{1} \times I_{2} \times I_{3} \times I_{4} \mid I_{2} \in A\left(\operatorname{Nil}\left(R_{2}\right)\right), I_{3} \in A\left(\operatorname{Nil}\left(R_{3}\right)\right), I_{4} \in A\left(\operatorname{Nil}\left(R_{4}\right)\right)\right\}, \\
C_{2}= & \left\{I_{1} \times R_{2} \times I_{3} \times I_{4} \mid I_{1} \in A\left(\operatorname{Nil}\left(R_{1}\right)\right), I_{3} \in A\left(\operatorname{Nil}\left(R_{3}\right)\right), I_{4} \in A\left(\operatorname{Nil}\left(R_{4}\right)\right)\right\}, \\
C_{3}= & \left\{I_{1} \times I_{2} \times R_{3} \times I_{4} \mid I_{1} \in A\left(\operatorname{Nil}\left(R_{1}\right)\right), I_{2} \in A\left(\operatorname{Nil}\left(R_{2}\right)\right), I_{4} \in A\left(\operatorname{Nil}\left(R_{4}\right)\right)\right\}, \\
C_{4}= & \left\{I_{1} \times I_{2} \times I_{3} \times R_{4} \mid I_{1} \in A\left(\operatorname{Nil}\left(R_{1}\right)\right), I_{2} \in A\left(\operatorname{Nil}\left(R_{2}\right)\right), I_{3} \in A\left(\operatorname{Nil}\left(R_{3}\right)\right)\right\}, \\
D= & \left\{I_{1} \times I_{2} \times I_{3} \times I_{4} \mid I_{1} \in A\left(\operatorname{Nil}\left(R_{1}\right)\right), I_{2} \in A\left(\operatorname{Nil}\left(R_{2}\right)\right), I_{3} \in A\left(\operatorname{Nil}\left(R_{3}\right)\right),\right. \\
& \left.I_{4} \in A\left(\operatorname{Nil}\left(R_{4}\right)\right)\right\} .
\end{aligned}
$$

If we put $A=\cup_{i=1}^{4} A_{i}, B=\cup_{i=1}^{6} B_{i}$ and $C=\cup_{i=1}^{4} C_{i}$, then one may check that $\{A, B, C, D\}$ is a partition of $V\left(A_{R}\right)$. We show that $A_{R}$ contains no induced odd cycle of length at least 5. Assume to the contrary, $a_{1}-a_{2}-\cdots-a_{n}-a_{1}$ is a induced odd cycle of length at least 5 in $A_{R}$. By Lemma 2.2, every vertex in $D$ is an isolated vertex in $A_{R}$ and thus $\left\{a_{1}, a_{2}, \ldots, a_{n}\right\} \cap D=\emptyset$. Next, we show that

$$
\left\{a_{1}, a_{2}, \ldots, a_{n}\right\} \cap C_{1}=\emptyset \text {. }
$$

To see this, if $a_{i} \in\left\{a_{1}, a_{2}, \ldots, a_{n}\right\} \cap C_{1}$, for some $1 \leq i \leq n$, then with no loss of generality, assume that $a_{1} \in C_{1}$. Since every vertex of $C_{1}$ is adjacent only to vertices of $A_{1}, a_{2}, a_{n} \in A_{1}$. This is impossible, as every vertex of $A_{R}$ is adjacent to $a_{2}$ if and only if it is adjacent to $a_{n}$. Therefore

$$
\left\{a_{1}, a_{2}, \ldots, a_{n}\right\} \cap C_{1}=\emptyset .
$$

Similarly,

$$
\left\{a_{1}, a_{2}, \ldots, a_{n}\right\} \cap C_{2}=\left\{a_{1}, a_{2}, \ldots, a_{n}\right\} \cap C_{3}=\left\{a_{1}, a_{2}, \ldots, a_{n}\right\} \cap C_{4}=\emptyset .
$$

Thus

$$
\left\{a_{1}, a_{2}, \ldots, a_{n}\right\} \cap C=\emptyset .
$$

Finally, we show that

$$
\left\{a_{1}, a_{2}, \ldots, a_{n}\right\} \cap B_{1}=\emptyset .
$$


Assume to the contrary and with no loss of generality, $a_{1} \in B_{1}$. As $a_{1}$ is adjacent only to vertices of $B_{2} \cup A_{3} \cup A_{4},\left\{a_{2}, a_{n}\right\} \subseteq B_{2} \cup A_{3} \cup A_{4}$. If $a_{2} \in B_{2}$, then $a_{3}$ is adjacent to $a_{n}$ (since if $a$ is adjacent to $a_{2}$ and $b$ is adjacent to $a_{1}, a$ is adjacent to $b)$, a contradiction. Thus $a_{2} \notin B_{2}$. Similarly, $a_{n} \notin B_{2}$ and so $\left\{a_{2}, a_{n}\right\} \subseteq A_{3} \cup A_{4}$. Since $A_{R}\left[A_{3} \cup A_{4}\right]$ is a complete bipartite graph, we conclude that $\left\{a_{2}, a_{n}\right\} \subseteq A_{3}$ or $\left\{a_{2}, a_{n}\right\} \subseteq A_{4}$. With no loss of generality, we may assume that $\left\{a_{2}, a_{n}\right\} \subseteq A_{3}$. This implies that $a_{3}$ is adjacent to $a_{2}$ and $a_{n}$ (since a vertex is adjacent to $a_{2}$ if and only if it is adjacent to $a_{n}$ ), a contradiction. Hence,

$$
\left\{a_{1}, a_{2}, \ldots, a_{n}\right\} \cap B_{1}=\emptyset .
$$

Similarly, for every $2 \leq i \leq 6$

$$
\left\{a_{1}, a_{2}, \ldots, a_{n}\right\} \cap B_{i}=\emptyset .
$$

This means that

$$
\left\{a_{1}, a_{2}, \ldots, a_{n}\right\} \subseteq A .
$$

But $A_{R}[A]$ is a complete 4 -partite graph with parts $A_{i}$ for $1 \leq i \leq 4$, a contradiction. Therefore, $A_{R}$ contains no induced odd cycle of length at least 5 and thus by Claim 1, Claim 2 and Theorem 3.1, we have $A_{R}$ is a perfect graph.

Acknowledgements. The authors thank to the referees for their careful reading and their excellent suggestions.

\section{REFERENCES}

[1] S. Akbari, A. Alilou, J. Amjadi and S. M. Sheikholeslami, The co-annihilating-ideal graphs of commutative rings, Canad. Math. Bull. 60 (2017), 3-11.

[2] D. F. Anderson and A. Badawi, On the total graph of a commutative ring without the zero element, J. Algebra Appl. 11 (2012), Article ID 1250074, 18 pages.

[3] D. F. Anderson and P. S. Livingston, The zero-divisor graph of a commutative ring, J. Algebra 217 (1999), 434-447.

[4] M. F. Atiyah and I. G. Macdonald, Introduction to Commutative Algebra, Addison-Wesley Publishing Company, Reading, Massachusetts, 1969.

[5] A. Badawi, On the annihilator graph of a commutative ring, Comm. Algebra 42 (2014), $108-121$.

[6] A. Badawi, On the dot product graph of a commutative ring, Comm. Algebra 43 (2015), 43-50.

[7] W. Bruns and J. Herzog, Cohen-Macaulay Rings, Cambridge University Press, Cambridge, 1997.

[8] R. Diestel, Graph Theory, Springer-Verlag, New York, USA, 2000.

[9] G. A. Dirac, On rigid circuit graphs, Abh. Math. Semin. Univ. Hambg. 38 (1961), 71-76.

[10] A. Mallika and R. Kala, A note on zero-divisor graph of amalgamated duplication of a ring along an ideal, AKCE Int. J. Graphs Comb. 14 (2017), 18-26.

[11] R. Nikandish, M. J. Nikmehr and M. Bakhtyiari, Coloring of the the annihilator graph of a commutative ring, J. Algebra Appl. 15 (2016), Article ID 1650124, 13 pages.

[12] T. T. Chelvam and K. Selvakumar, On the connectivity of the annihilating-ideal graphs, Discuss. Math. Gen. Algebra Appl. 35 (2015), 195-204.

[13] D. B. West, Introduction to Graph Theory, 2nd ed., Prentice Hall, Upper Saddle River, 2001. 
${ }^{1}$ Department of Mathematics,

Karaj Branch, Islamic Azad University, KARAJ, IRAN.

Email address: m.saadat82m@yahoo.com

${ }^{2}$ Faculty of Mathematics,

K. N. Toosi University of Technology, TEHRAN, IRAN.

Email address: nikmehr@kntu.ac.ir

${ }^{3}$ Department of Basic Sciences, Jundi-Shapur University of Technology, DEZFUL, IRAN.

Email address: r.nikandish@jsu.ac.ir 